\title{
Implementación de una estrategia didáctica en la Clínica de Odontopediatría, Facultad de Odontología, UCR
}

\section{Implementation of a didactic strategy at the UCR Dentistry Faculty}

\author{
Natalia Gutiérrez Marín \\ Facultad de Odontología, Universidad de Costa Rica \\ San José, Costa Rica \\ natalia.gutierrez@ucr.ac.cr
}

Recibido: 04-VI-2012 • Aceptado: 26-VIII-2012 • Corregido: 26-XI-2012

\begin{abstract}
Resumen: El presente trabajo muestra la implementación de una estrategia didáctica: el método de casos mediados con las Tecnologías de la Información y Comunicación (TIC), específicamente el uso del blog, con un grupo de estudiantes del II semestre del 2010 que asisten a la clínica de Odontopediatría de la Facultad de Odontología, Universidad de Costa Rica (UCR). El objetivo consistió en mejorar en el estudiante, mediante el uso de la estrategia, sus habilidades diagnósticas para indicar un adecuado tratamiento dental. Como resultados, se observó que la estrategia empleada produjo buenos efectos en el aprendizaje y su empleo promovió un buen nivel de satisfacción en los estudiantes. Se recomienda aplicarlo en un grupo mayor para verificar su eficacia.
\end{abstract}

Palabras clave: Estrategia didáctica, método del caso, TIC, diagnóstico y tratamiento dental.

Abstract: This article describes the implementation of a didactic strategy: the case method using Information and Communication Technology (ICT), specifically the use of blogs with a group of pedodontic students from the Universidad de Costa Rica (UCR), Faculty of Dentistry during the second semester of 2010. The objective was to improve the student's diagnostic skills when

\section{Introducción}

En la actualidad, los y las estudiantes que ingresan a la Clínica de Odontopediatría de la Universidad de Costa Rica (UCR), presentan deficiencias en lo que se refiere al diagnóstico y planeamiento del tratamiento dental. Esto se debe a que, en los años previos, han recibido muchos conocimientos teóricos, pero no han tenido la oportunidad de integrarlos en un contexto real.

Tradicionalmente, la dinámica de la clínica es que el estudiante realiza una anamnesis, un examen clínico y radiográfico del paciente que va a atender y con esa información, en su casa, elabora una presentación del caso que luego expondrá al instructor a cargo.

Cuando el discente realiza dicha presentación es donde se evidencia la 
suggesting a proper dental treatment. As a result, the strategy produced good effects in learning and the student's satisfaction levels were good too. It is highly recommended to apply this strategy with a bigger audience to confirm/validate its effectiveness.

Keywords: Didactic strategy, case method, ICT, diagnostic and dental treatment. deficiencia antes mencionada $\mathrm{y}$, en ese momento, el instructor lo que utiliza como estrategia didáctica para reforzar las habilidades diagnósticas y el planeamiento dental es enviar al estudiante a que profundice en el tema. El discente por su parte, lo que generalmente hace es recurrir a sus apuntes de años anteriores, los cuales son datos teóricos que no contribuyen a superar la carencia detectada. El Departamento de odontopediatría no cuenta con un material de apoyo visual que contribuya a solventar esta situación.

Por lo anterior, es que se plantea la utilización de una estrategia didáctica como lo es el método del caso en conjunto con una tecnología de la información y comunicación, en este caso en particular con la utilización de un blog. El objetivo general es mejorar en el estudiante, mediante el uso de casos clínicos y una TIC (blog), sus habilidades diagnósticas para indicar un adecuado tratamiento dental. Dentro de los objetivos específicos están: agudizar la observación clínica para identificar caries o alguna patología en particular, analizar radiografías para observar caries incipientes, patologías radiculares o hallazgos relevantes, e integrar la observación clínica y radiográfica para plantear el tratamiento más indicado.

El aprendizaje es un proceso a través del cual se adquieren nuevas habilidades, destrezas, conocimientos, conductas o valores como resultado del estudio, la experiencia, la instrucción, el razonamiento y la observación (Cardozo, 2008). Debido a lo anterior, las estrategias didácticas constituyen una herramienta útil en el proceso de aprendizaje.

Las estrategias didácticas son recursos utilizados por el docente para promover el aprendizaje. El término estrategia hace referencia a que el docente o estudiante deben emplearlas como procedimientos flexibles y adaptativos a distintas circunstancias de enseñanza (Díaz, Hernández y Barriga, 2002). Dentro de dichas estrategias encontramos: el aprendizaje basado en 
problemas, orientado a proyectos, colaborativo y el método del caso que es al que se va a hacer referencia.

El método del caso, también llamado análisis o estudio de casos, como técnica de aprendizaje tuvo su origen en la Universidad de Harvard (aproximadamente en 1914), con el fin de que los estudiantes de Derecho, en el aprendizaje de las leyes, se enfrentaran a situaciones reales y tuvieran que tomar decisiones, valorar actuaciones y emitir juicios fundamentados. Con el paso de los años el método de caso fue extendiéndose a otros contextos y estudios, y se convirtió en una estrategia muy eficaz para que los estudiantes adquieran diversos aprendizajes y desarrollen diferentes habilidades gracias al protagonismo que tienen en la resolución de los casos (Heinsen, 2009).

Debido a las características propias de la enseñanza de la odontología, se escoge el método del caso ya que es una técnica de aprendizaje activa, centrada en la investigación del estudiante sobre un problema real y específico que ayuda al alumno a adquirir la base para un estudio inductivo. Parte de la definición de un caso concreto para que el estudiantado sea capaz de comprender, de conocer y de analizar todo el contexto y las variables que intervienen en el caso.

Aunado a las estrategias didácticas, los procesos de aprendizaje se pueden favorecer de las tecnologías de la información y comunicación (TIC), que se definen como el conjunto de instrumentos tecnológicos que permiten la adquisición, producción, almacenamiento, tratamiento, comunicación, registro y presentación de informaciones contenidas en señales de naturaleza acústica (sonidos), óptica (imágenes) o electromagnéticas (datos alfa numéricos) (Cardozo, 2008).

Según Cabero (2008), existen las TIC tradicionales y las nuevas TIC. Las TIC tradicionales son aquellas herramientas comunicacionales utilizadas antes de la aparición del Internet; algunas de ellas son: la voz, el papel impreso, los sonidos, la radio, la televisión y los videos. Estas técnicas son muy usadas en ciertas áreas rurales donde las facilidades tecnológicas y los recursos económicos son limitados.

Las nuevas TIC son procesos y productos derivados de las nuevas herramientas informativas a saber: software, hardware e Internet. Entre sus ventajas se citan: la interactividad, la instantaneidad, altos parámetros en cuanto a calidad de imagen y de sonido y la digitalización. Pero a la vez presentan una gran desventaja que consiste en que la accesibilidad es limitada siempre y cuando no se rompa la brecha digital que existe entre zonas rurales y urbanas (Pedró y Benavides, 2007).

Tanto las estrategias didácticas como las TIC son herramientas útiles para trasmitir los conocimientos a los estudiantes, quienes deben tener muy claras las bases teóricas respecto a la caries, su diagnóstico y su tratamiento para poder desenvolverse en un futuro en el entorno laboral.

Uno de los conceptos teóricos fundamentales que debe tener el alumnado de odontología es el de la caries dental, la cual es un proceso que implica un desequilibrio de las interacciones moleculares normales entre la superficie/subsuperficie del diente y el biofilm microbiano adyacente. Este desequilibrio se manifiesta en un cierto plazo como desmineralización acumulativa del diente que, si es desenfrenado, tiene el potencial de producir cavitación del esmalte y daño colateral a la dentina y a la pulpa, culminando con la destrucción localizada de los tejidos duros del diente (Villalobos, Medina, Vallejos y Espinoza, 2005).

La caries es un proceso dinámico que muestra modificaciones continuas por acción de fluoruros, hábitos de higiene y dieta, lo que la convierte en un proceso patológico activo, donde el odontólogo debe estar preparado para brindar información, motivación, esfuerzo y orientación en conducta, higiene y salud oral (Rivera, Martínez y Hernández, 2006). 
El diagnóstico de la caries puede determinarse principalmente por tres procedimientos: exploración clínica, exploración radiográfica o transiluminación.

En la exploración clínica, los hallazgos serán diferentes en función de la fase en que se encuentre la enfermedad, y se pueden observar desde cambios de coloración en las lesiones incipientes hasta cavidades en esmalte y dentina en lesiones severas. En la exploración clínica se puede incluir:

La inspección visual se efectúa con los dientes limpios y secos. Se puede realizar directamente o si se precisa, se pueden utilizar espejos o lentes de aumento.

En la exploración táctil se intenta detectar la cavitación o reblandecimiento del esmalte. Se debe ejecutar con mucho cuidado para evitar dañar el esmalte intacto.

En la exploración radiográfica, las imágenes radiográficas se producen por la diferente capacidad (densidad) que tienen los tejidos de atenuar los rayos X. El esmalte y la dentina (cristales de hidroxiapatita con gran contenido inorgánico) atenúan mucho los rayos $\mathrm{X}$, dando lugar a una imagen blanquecina en la radiografía. En cambio, la pulpa (tejido conectivo con gran contenido orgánico) atenúa poco los rayos $\mathrm{X}$, provocando una imagen gris oscura en la radiografía. A la hora de evaluar la caries mediante una radiografía, se tiene que tener en cuenta que se está observando únicamente aquellas zonas de desmineralización que producen cambios en la absorción de los rayos $\mathrm{X}$, pudiendo existir caries que no se detectan. Además, tienen el inconveniente de que son imágenes en dos dimensiones que representan un objeto de tres dimensiones (Barbiere, Flores, Escribano y Discepoli, 2006).

Por último, la transiluminación es un método que se basa en el hecho de que el esmalte de las lesiones cariosas tiene un índice de trasmisión de la luz menor que el del esmalte sano. Utilizando una luz, preferiblemente brillante para iluminar el diente, las caries aparecerán más oscuras ya que la luz es absorbida en mayor cantidad cuando se encuentra una lesión desmineralizada (Rubio, Cueto, Suarez y Frieyro, 2006).

\section{Diseño de la estrategia didáctica}

\section{Fundamentación teórica}

Se seleccionó el método del caso debido a que muchas de sus ventajas ayudan al objetivo planteado. Dentro de las ventajas se encuentran: facilita la comprensión de los problemas divergentes y la adopción de soluciones mediante la reflexión y el consenso, mejora la retención de la información $\mathrm{y}$ el conocimiento adquirido al discutir y practicar acerca de los conceptos utilizados, refuerza el aprendizaje significativo, ya que el alumno usa conocimientos previos para el análisis del problema y la propuesta de soluciones facilitando la generación de los conceptos usados; fortalece la resolución de problemas, genera conocimientos y promueve la creatividad, y por último, aumenta la motivación del alumnado por el tema de estudio al confrontarle con situaciones relativas al ejercicio de la profesión. Las situaciones de aula son más motivadoras y dinámicas ya que facilitan una mejor asimilación de los conocimientos (Vargas, 2009).

Asimismo, se escogió la implementación del blog ya que los y las estudiantes tienen poco tiempo para actividades extra fuera de sus planes de estudios y el blog no demanda horarios específicos o desplazarse a la universidad.

\section{Descripción de la estrategia}

La estrategia didáctica que se utilizó consistió en el empleo de casos clínicos dentro de un blog creado para tal fin (casosclinicosodontopediatria.blogspot.com).

Para la creación de esta estrategia didáctica, se incluyó la información de los 
mismos pacientes que asisten a la Clínica de Odontopediatría de la Facultad de Odontología de la UCR y que son atendidos por los estudiantes. A los pacientes se les hizo un examen clínico y una vez definidas las piezas que se iban a utilizar en la estrategia, se tomaron fotografías y radiografías de dichas piezas (lo anterior se hizo previa autorización de los padres y tuvo una duración de dos semanas). Posteriormente, se fotografiaron las respectivas radiografías. No se utilizó la transiluminación porque no es método de rutina para detectar caries empleado en la Facultad.

Luego, con el material recolectado, se elaboraron cinco casos clínicos donde en cada uno se daba una explicación general del paciente y de la pieza que se quería analizar, cada caso se acompañó de una fotografía clínica y otra de la radiografía, lo anterior se hizo en una semana.

El blog (creado en dos días) consta de varias partes. Primero, hay un encabezado donde se da una explicación del propósito del mismo y se indica que la actividad a realizar es analizar los casos clínicos planteados y dar un diagnóstico y tratamiento adecuado. Para esto se señaló un espacio donde cada estudiante colocara sus respuestas.

La segunda parte es el cuerpo del blog, donde están los cinco casos a analizar. Los y las estudiantes tuvieron una semana para responder a dichos casos.

La tercera parte es una encuesta para valorar la utilidad del blog. Esta encuesta consta de tres preguntas: la primera pregunta fue: "¿Considera usted que este blog le fue útil para mejorar sus habilidades diagnósticas?" y las opciones de respuestas eran: mucho, poco, nada; la segunda pregunta fue: "¿Cada cuánto le gustaría que este blog se actualizara?" y las opciones de respuesta eran: semanalmente, quincenalmente o mensualmente; y la tercera pregunta fue: “¿Cuántos casos clínicos cree conveniente que estén en cada entrada del blog?" y sus respectivas opciones fueron cada: $3,5,8$ o 10 .
Una vez que el estudiantado respondiera a los casos clínicos, se incluirían en el blog los diagnósticos y tratamientos más acertados para que los y las estudiantes revisaran y respondieran si así lo consideraban (para esto se contó con una semana).

Para evaluar la estrategia, además de la encuesta que se incluyó en el blog, una vez que el alumnado hubiera participado (15 días posteriores al inicio de la activación del blog), se les reunió para comentar sobre la experiencia realizada.

\section{Papel del docente}

Respecto al papel docente, este fue el encargado de recolectar la información, plantear los casos clínicos y crear el blog como primera acción. Una vez que los estudiantes postearon sus respuestas en la sección de comentarios del blog, se incorporó los diagnósticos y tratamientos más acertados en el contexto en que se planteaba cada caso.

La parte más importante fue la que correspondió a la motivación que se dio al estudiantado para que participaran de la estrategia. Dicha participación no medió calificación alguna, en primera instancia porque el curso es colegiado y no se pueden hacer cambios una vez que este haya empezado y se les haya entregado el programa (como sucedió en este caso), y, por otra parte, porque en realidad la intención no es que los estudiantes realicen cierta actividad solo con el fin de obtener una calificación, sino por el deseo de adquirir conocimientos que les resulten de mucha utilidad en sus futuras prácticas profesionales.

\section{Papel del estudiantado}

El papel del alumnado fue activo. Cada uno de los y las estudiantes ingresó al blog y respondió a los casos planteados. Además, todos participaron de las 
preguntas de la encuesta que valoraban la estrategia utilizada.

Una vez concluida la estrategia, los discentes participaron en una pequeña reunión que se hizo para expresar sus aportes y las mejoras que creían convenientes para optimizar el blog. Esto se expondrá mas adelante en la sección de valoración de los resultados.

\section{Papel del contexto}

El curso se imparte en un área clínica que tiene una oficina anexa que cuenta con una computadora de escritorio pero no cuenta con conexión a Internet. Tanto la cámara fotográfica para recolectar las fotografías para los casos clínicos, como la computadora portátil con acceso a Internet, fueron suministradas por el docente. Los y las estudiantes harían uso del blog ya fuera en sus casas o en una sala con acceso a Internet en el segundo piso de la Facultad.

Aplicación de la estrategia didáctica

Una vez creado el blog, se reunió a los 5 estudiantes participantes y se les explicó la dinámica del mismo: se les mostró mediante la computadora cómo deberían ingresar, y cómo sería la actividad que realizarían. Además, se les entregó una tarjeta con la dirección de blog para que no hubiera omisiones en la participación por olvido de la misma.

Se les indicó que el blog estaría activo por una semana y, posterior a esto, se incluirían los diagnósticos y tratamientos más indicados para que el estudiantado revisara, analizara y comparara con sus propios aportes, para esto tendrían una semana más por si querían agregar algo.

\section{Valoración de los resultados}

Las respuestas dadas a los casos clínicos respecto a los diagnósticos y planes de tratamiento fueron acertadas, ya que los discentes emitieron un juicio apropiado de cada situación planteada y a la vez trazaron los pasos a seguir para solucionar cada problema propuesto.

Respecto a la encuesta planeada en el blog, a la pregunta: “¿Considera usted que este blog le fue útil para mejorar sus habilidades diagnósticas?", 4 estudiantes respondieron mucho, 1 respondió poco y la opción nada no fue escogida. En la siguiente pregunta: “¿Cada cuánto le gustaría que este blog se actualizara?", 1 estudiante respondió que semanalmente, otro quincenalmente y 3 indicaron que mensualmente. Con respecto a la última pregunta: “¿Cuántos casos clínicos cree conveniente que estén en cada entrada del blog?", 3 participantes indicaron que 5 casos y los restantes 2 opinaron que 10 casos, ningún estudiante señaló ni 3 ni 8 casos.

Luego de que se incluyeron los diagnósticos y tratamientos más indicados, no hubo aportes o comentarios de los y las estudiantes, probablemente porque los diagnósticos de ellos no discrepaban significativamente de las respuestas dadas.

\section{Bondades}

Dentro de las bondades encontradas respecto a la estrategia, se puede citar que al ser un grupo muy pequeño, es más fácil el control y, por ende, la participación es más segura.

Además, los blog resultan ser una herramienta de muy fácil manipulación, máxime que al parecer el estudiantado posee muchas habilidades con el manejo de programas de cómputo y afines.

De acuerdo con Cabero (2008), la utilización del blog rompe con el modelo unidireccional de formación y con la exigencia de que el profesor esté presente en una aula, lo que lo convierte en un buen aporte al sistema educativo.

Aunado a lo anterior, también se puede citar como bondad el hecho que no se requiere establecer un horario rígido para realizar la estrategia, como sucedería si no 
se utilizaran TIC y los casos se mostraran de otra forma.

Otro aspecto positivo es que los y las estudiantes analizaron fotografías de pacientes reales, en los que efectuarían posteriormente los tratamientos; esto hace que se enfrenten a situaciones que no cumplen con todo lo establecido tal y como se presentan en la literatura, sino que pueden tener muchas variantes como en la vida real.

\section{Limitaciones}

Una de las limitaciones que tuvo la aplicación de la estrategia fue que no se dio una retroalimentación, ya que los casos eran planteados rígidamente (aunque con la mayor claridad posible) y la herramienta utilizada: el blog, no dio oportunidad para que el estudiantado interaccionara con el docente para aclarar alguna duda o ampliar algún concepto.

Otra limitación que se encontró es que los y las estudiantes que participaban podían observar las respuestas posteadas por los compañeros que habían ingresado antes que ellos, por lo que no se sabe si esos comentarios influyeron en las respuestas posteriores.

En el grupo que se aplicó el blog no hubo problemas con el uso de la tecnología, pero en otros contextos, donde el estudiantado no estuviera familiarizado con el uso de los blogs o donde el alumnado no contara con facilidades para utilizar equipos de cómputo con la conectividad a Internet requerida; el uso de TIC podría ser una limitante.

\section{Proyecciones de uso}

La Clínica de Odontopediatría consta de aproximadamente 60 estudiantes cada semestre, así que debido a los buenos resultados que obtuvo la estrategia, se recomienda implementarla en el grupo completo, primero se podría utilizar los mismo casos planteados y según los resultados, se podrían incluir muchas otras áreas del aprendizaje como lo pueden ser: valoraciones de restauraciones previas, cómo actuarían ante situaciones de emergencia como lo es la fractura de una pieza dental o rehabilitaciones completas entre otros.

\section{Valoración de la estrategia desde la perspectiva del estudiante}

Desde el punto de vista del alumnado, respondieron acertadamente en los casos clínicos y estuvieron bastante satisfechos con la experiencia, eso lo demostraron en la encuesta tanto como en la reunión posterior. Uno de los estudiantes señaló: "es algo nuevo que nos puede servir para diagnosticar caries".

Si indicaron la posibilidad de integrarlo a foros o sitios similares para que sea algo mas interactivo, a lo anterior se dijo: "sería bueno combinarlo con redes sociales o foros".

También exteriorizaron: "en los cursos clínicos no se nos ofrecen espacios como este blog, siempre se reduce a aprender practicando sin soportes adicionales".

\section{Valoración de la estrategia desde la perspectiva del docente}

La estrategia utilizada fue bastante acertada, sobretodo tomando en cuenta que es un curso clínico donde los trabajos colaborativos, visitas u otro tipo de actividades no serían pertinentes.

Se logra el objetivo de desarrollar en los y las estudiantes habilidades diagnósticas en un contexto real, ya que ellos tuvieron la oportunidad de analizar en el blog imágenes clínicas y radiográficas de distintas situaciones dentales, donde, aplicando los conocimientos teóricos adquiridos previamente, lograron emitir tanto una adecuada valoración de cada caso propuesto como un correcto planteamiento del tratamiento más indicado. 


\section{Conclusiones}

El aplicar nuevas estrategias didácticas al curso trae un componente innovador que resultó interesante y bien aprovechado por los discentes. A parte que, compromete a los y las docentes a estar a la vanguardia de las nuevas tendencias educativas donde los estándares de calidad de la enseñanza, las metodologías, los avances en la evaluación del conocimiento y de las competencias adquiridas alcanzan gran protagonismo.

La utilización del método del caso en conjunto con el blog logró que los y las estudiantes mejoraran sus habilidades diagnósticas tanto en la identificación de caries como en el análisis radiográfico y en el planteamiento del tratamiento más adecuado.

Asimismo, el uso de TIC en el área de la odontopediatría podría propiciar el trabajo en equipo, ya que el alumnado al utilizar el blog o más aún, si se incorporara un chat o el uso de wikis, podrían interaccionar entre ellos debatiendo algún caso en particular donde el diagnóstico y plan de tratamiento no sea tan evidente y estructurado como lo puede plantear un libro.

\section{Referencias}

Barbiere, G.; Flores, J.; Escribano, M., y Discepoli, N. (2006). "Actualización en radiología dental. Radiología convencional vs radiología digital". Avances en odontoestomatología. 22 (2), 131-139.

Cabero, J. (2008) "Nuevas tecnologías, comunicación y educación”. Revista Comunicar. (3), 14-25. Recuperado de http://tecnologiaedu.us.es/revistaslibros/12.htm
Cardozo, G. (2008). "Tecnologías de la información y la comunicación en la educación”. Revista Iberoamericana de Educación. No. 45/1-25.

Díaz, F.; Hernández, G., y Barriga, A. (2002). Estrategias docentes para un aprendizaje significativo y una interpretación constructivista. II Ed. México: Editorial Mc Graw Hill.

Heinsen, M. (2009). "Método de casos". Unibe informa. Recuperado de www. unibe.edu.do

Pedró, F., y Benavides, F. (2007). "Políticas educativas sobre nuevas tecnologías en los países iberoamericanos". Revista Iberoamericana de Educación. Recuperado de www. rieoei.org/45justificacion

Rivera, G.; Martínez, J., y Hernández, E. (2006). "Caries dental e higiene bucal en adolescentes". Revista Asociación Dental Mexicana. (6), 231-234.

Rubio, E.; Cueto, M.; Suarez, M., y Frieyro, J. (2006). "Técnicas de diagnóstico de la caries dental. Descripción, indicaciones y valoración de su rendimiento". Boletín de la sociedad de pediatría de Asturias, Cantabria, Castilla y León. (46), 23-31.

Vargas, C. (2009). "El método del caso en la enseñanza del derecho: experiencia piloto de un piloto novel". Revista de formación e Innovación Educativa Universitaria. 2 (4), 193-206.

Villalobos, J.; Medina, C.; Vallejos, A., y Espinoza, J. (2005). "Caries dental en escolares de 6 a 12 años de Navolato, Sinaba: resultados preliminares". Revista Biomédica. 16 (3), 217-219. 